\title{
Wirtszellreaktivierung demisch induzierter Letalschäden im DNS-haltigen Serratia-Phagen Kappa
}

\author{
ULRICH WINKLER \\ Institut für Mikrobiologie, J. W. Goethe Universität, Frankfurt/Main \\ (Z. Naturforschg. 20 b, 864—867 [1965] ; eingegangen am 17. März 1965)
}

\begin{abstract}
After treating free phage Kappa with nitrous acid, triethylenemelamine, ethylmethanesulfonate, or hydroxylamin and using these phages for infecting Serratia HY wildtype cells, at least $20 \%$ of the lethal damage in the phage-DNA can be reactivated by the host (= host cell reactivation). It is known that all lethal agents tested so far attack the primary structure of the DNA in different ways. Therefore, we assume that the target for the host cell reactivation consists of some damage in the secondary structure of the DNA, because there is probably some coincidence in the action of all agents. The hypothesis that in the DNA changes of thymine are a prerequisite for host cell reactivation has been disproved by the experiments with nitrous acid and ethylmethanesulfonate because both substances do not act on thymine.
\end{abstract}

Mit einer modifizierten Stempel-Technik ${ }^{1}$ wurden von Serratia marcescens, Stamm HY, mehrere sog. hcr-Mutanten * isoliert, die sich folgendermaßen vom Wildtyp unterscheiden: a) Ihre Fähigkeit zur Koloniebildung ist UV-sensibler; b) Extrazellulär UV-bestrahlter Phage Kappa überlebt auf ihnen schlechter als auf dem Wildtyp ${ }^{1}$.

Mutanten dieses Verhaltens wurden schon früher von Escherichia coli isoliert ${ }^{2}$. Im Gegensatz zum Wildtyp sind sie zur sogen. Wirtszellreaktivierung (HCR) * unfähig, das heißt in der DNS von UVbestrahlten Zellen können Letalschäden enzymatisch ohne Licht nicht mehr beseitigt werden ${ }^{3-7}$.

Röntgenbestrahlter Phage Kappa überlebt auf Serratia HY Wildtyp besser als auf der Mutante hcr42, das heißt (außer UV-Schäden) ist auch ein Teil (20\%) der durch Röntgenstrahlen induzierten Letalschäden in der DNS von Kappa Wirtszell-reaktivierbar ${ }^{1}$. Sofern man die HCR als einen streng Schadenspezifischen Vorgang betrachtet, wäre daraus zu folgern, daß UV- und Röntgenstrahlen mindestens ein für beide gemeinsames, letal wirksames Bestrahlungsprodukt in der DNS bilden. Es könnte aber

\footnotetext{
* host cell reactivation.

* Diese Arbeit ist ein Teil der Habilitationsarbeit, die vom Verf. bei der Naturwissenschaftlichen Fakultät der J. W. Goethe-Universität in Frankfurt/Main im Sommer 1965 eingereicht wurde.

1 U. WinkLer, Virology 24, 518 [1964].

2 U. WinkLer, Anreicherungskultur und Mutantenauslese; Sympos. Göttingen 1964, Gustav Fischer Verlag, Stuttgart, im Druck.

${ }^{3}$ R. P. Boyce u. P. Howard-Flanders, Proc. nat. Acad. Sci. USA 51, 293 [1964].

4 D. Pettijohn u. P. Hanawalt, J. molecular Biol. 9, 395 [1964].
}

auch sein, daß mehr als eine Art von DNS-Schaden Wirtszell-reaktivierbar ist. Wahrscheinlich trifft letztere Annahme zu; denn im folgenden wird die HCR von Letalschäden im Phagen Kappa gezeigt, die durch vier verschiedenartig wirkende Chemikalien ausgelöst wurden. Dieses Resultat sagt aber selbstverständlich nichts darüber aus, ob UV- und Röntgenstrahlen wenigstens z. T. identische letale Bestrahlungsprodukte in DNS bilden können oder nicht.

\section{Material und Methoden}

Es wurde mit dem temperierten Phagen Kappa ${ }^{8}$ gearbeitet, der doppelsträngige DNS enthält ${ }^{9}$ sowie dem Kappa-sensiblen Bakterien-Stamm HY ${ }^{10}$ von Serratia marcescens und drei hiervon isolierten Mutanten ${ }^{1}$ hcr42, hcr64 und hcr91, deren Fähigkeit zur HCR unterschiedlich ist.

Die allgemeine Versuchstechnik entsprach der von Adams ${ }^{11}$ beschriebenen. Als Grundagar wurde 1,5-proz. und als Gußschicht 0,5-proz. Bouillonagar (DIFCO) mit $0,5 \% \mathrm{NaCl}$ benutzt. Bei $30^{\circ} \mathrm{C}$ gerollte gesättigte Übernachtkulturen der Bakterien in Bouillon (DIFCO) mit $0,5 \% \mathrm{NaCl}$ dienten als Indikator für den Phagen Kappa. Dieser war in einer farblosen Mutante von

5 A. Rörsch, C. Van Der Kamp u. J. Adema, Biochim. biophysica Acta [Amsterdam] 80, 346 [1964].

6 R. B. Setrow u. W. L. Carrier, Proc. nat. Acad. Sci. USA 51, 226 [1964].

7 R. C. Shuster u. R. P. Boyce, Biochem. biophysic. Res. Commun. 16, 489 [1964].

8 H. Ellmauer u. R. W. Kaplan, Naturwissenschaften 46, 150 [1959].

${ }^{9}$ F. W. Pons, in Vorbereitung.

10 E. L. Labrum u. M. I. Bunting, J. Bacteriol. 65, 394 [1953].

11 M. H. Adams, Bacteriophages, Interscience Publ., Inc., New York, pp. 592 [1959]. 
Serratia HY auf Gußplatten vermehrt worden. Nach Filtrieren durch 3G5M-Filter (Schott \& Gen.) und nieder- und hochtourigem Zentrifugieren wurde er gereinigt in $\mathrm{H}$ ersh e y-Puffer kühl aufgehoben $\left(1,6 \cdot 10^{11} / \mathrm{ml}\right)$. Alle Verdünnungen bis auf eine Ausnahme (s.u.) wurden in Puffersaline der folgenden Zusammensetzung durchgeführt: $4 \mathrm{~g} \mathrm{NaCl}, 7 \mathrm{~g}$ $\mathrm{Na}_{2} \mathrm{PHO}_{4} \cdot 2 \mathrm{H}_{2} \mathrm{O}, 3 \mathrm{~g} \mathrm{KH}_{2} \mathrm{PO}_{4}, 2 \mathrm{ml}$ einer 1-m. Lösung von $\mathrm{MgSO}_{4} \cdot 7 \mathrm{H}_{2} \mathrm{O}, 1000 \mathrm{ml}$ dest. Wasser.

Die chemische Inaktivierung des Phagen Kappa erfolgte im wesentlichen nach Angaben von Beckmann ${ }^{12}$ :

Triäthylenmelamin $=$ TEM (Hoechst AG) : $\mathrm{Zu} \mathrm{9,9} \mathrm{ml}$ einer 0,01-proz. Lösung in Puffersaline (ohne $\mathrm{MgSO}_{4}$ ) wurde $0,1 \mathrm{ml}$ unverdünnte Phagen-Suspension pipettiert. Das Reaktionsgemisch $\left(p_{\mathrm{H}} 7,0\right)$ wurde bei $37{ }^{\circ} \mathrm{C}$ gehalten.

Hydroxylaminhydrochlorid $=H A$ (E. Merck AG; Nr. 4616): $\mathrm{Zu} \mathrm{9,9} \mathrm{ml} \mathrm{einer} \mathrm{1-m.} \mathrm{Lösung} \mathrm{in} \mathrm{Puffer-}$ saline (ohne $\mathrm{MgSO}_{4}$ ), die durch Zugabe von 10-n. $\mathrm{NaOH}$ auf $p_{\mathrm{H}} 7,5$ eingestellt worden war, wurde $0,1 \mathrm{ml}$ unverdünnte Phagen-Suspension pipettiert. Das Reaktionsgemisch wurde bei $37^{\circ} \mathrm{C}$ gehalten und die Proben ausnahmsweise in Dilution BRотн ${ }^{13}$ verdünnt.

Äthylmethansulfonat = EMS (Centre d'études du Bouchet, Frankreich): $\mathrm{Zu} 19,8 \mathrm{ml}$ von $0,5-m$. Acetatpuffer vom $p_{\mathrm{H}} 4,62$ (E. Merck AG) wurden $0,2 \mathrm{~m}$ ] EMS pipettiert. Mii $10-n$. $\mathrm{NaOH}$ wurde das $p_{\mathrm{H}}$ auf 6,6 eingestellt. Dann wurden 4,9 ml dieser Lösung mit $0,1 \mathrm{ml}$ unverdünnter Phagen-Suspension vermischt und bei $37{ }^{\circ} \mathrm{C}$ gehalten.

Natriumnitrit (E. Merck AG; Nr. 6544) : Zu 3,7 ml von 1-m. Acetatpuffer vom $p_{\mathrm{H}}$ 4,62 wurden nacheinander 1,25 ml 10-proz. Essigsäure, 0,1 ml unverdünnte

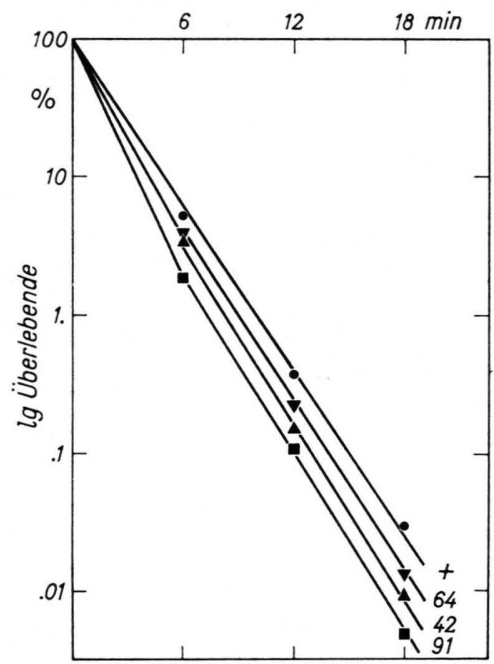

Abb. 1. Überleben des Phagen Kappa nach verschieden langer Behandlung mit Nitrit. $+=$ Serratia HY Wildtyp; 42, 64 und $91=$ hcr- $^{-}$Mutanten von Serratia $\mathrm{HY}$.

12 H. Beckmann, Diss., Naturwiss. Fakultät, Universität Frankfurt/M., pp. 86 [1964] ; H. Beckmann u. R. W. Kaplan, Z. allg. Mikrobiol. 5, 1 [1965].

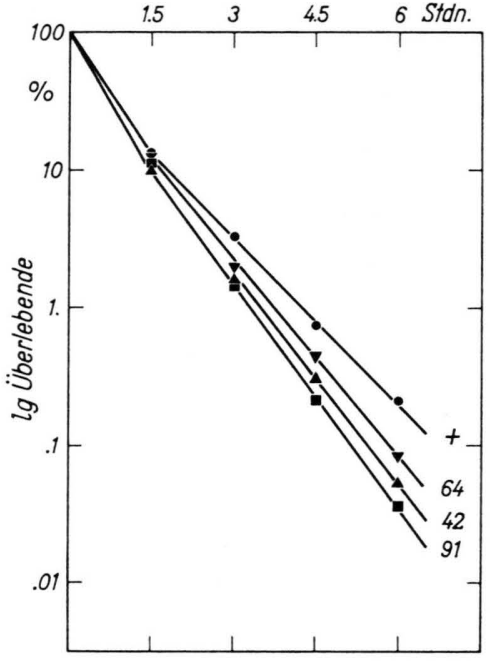

Abb. 2. Überleben des Phagen Kappa nach verschieden langer Behandlung mit Triäthylenmelamin (Erklärung s. Abb.1).

\begin{tabular}{|c|c|c|c|c|}
\hline \multirow{2}{*}{$\begin{array}{c}\text { Einwirkung } \\
\text { von HA } \\
\text { [Stdn.] }\end{array}$} & \multicolumn{4}{|c|}{ Überleben von Kappa [\%] auf } \\
\hline 12 & hcr $^{+}$ & hcr42 & hcr64 & hcr91 \\
\hline 24 & 8,0 & 9,9 & 7,9 & 2,4 \\
\hline
\end{tabular}

Tab. 1. Überleben des Phagen Kappa nach 12- und 24-stdg. Behandlung mit Hydroxylamin.

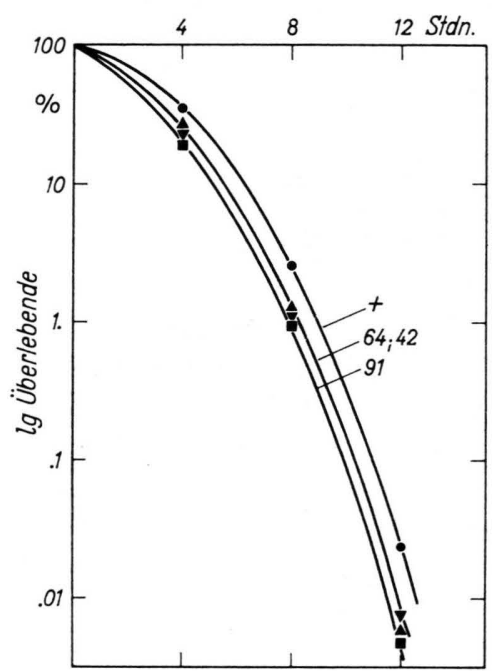

Abb. 3. Uberleben des Phagen Kappa nach verschieden langer Behandlung mit Äthylmethansulfonat (Erklärung s. Abb. 1).

13 E. Freese, E. Bautz-Freese u. E. Bautz, J. molecular Biol. 3,133 [1961]. 
Phagen-Suspension und $0,05 \mathrm{ml}$ einer $1,25-m$. $\mathrm{NaNO}_{2}$ Lösung pipettiert. Das Reaktionsgemisch ( $p_{\mathrm{H}}$ ca. 4,1) wurde in einem Wasserbad bei $30^{\circ} \mathrm{C}$ gehalten, in dem schon der Acetatpuffer und die Essigsäure vor der Phagenzugabe vorgewärmt worden waren.

Nach verschieden langer Zeit der chemischen Behandlung des freien Phagen wurden Proben verdünnt und parallel auf Serratia HY Wildtyp und den Mutanten hcr42, hcr64 und hcr91 ausgeplattet. Nach etwa 18-stdg. Bebrütung der Platten bei $30{ }^{\circ} \mathrm{C}$ wurden die Plaques ausgezählt. Alle Versuchspunkte in den Abbildungen und der Tabelle sind das geometrische Mittel von zwei Experimenten. Vorversuche ergaben ähnliche Ergebnisse; die Streuung zwischen den Versuchen war gering.

\section{Ergebnisse}

Extracellulär mit Nitrit, Triäthylenmelamin (TEM) oder Äthylmethansulfonat (EMS) behandelter Phage Kappa hat auf den Mutanten hcr42, hcr64 und hcr9l eine geringere Chance zum Überleben als auf dem zugehörigen Wildtyp (Abb. 1-3). Das bedeutet, daß wahrscheinlich verschiedenartige Letalschäden in der Primärstruktur der DNS von Kappa Wirtszell-reaktivierbar sind; denn bisher kennt man keine chemische Veränderung von Bausteinen der DNS, die sowohl durch UV- und Röntgenstrahlen als auch durch Nitrit, TEM und EMS ausgelöst werden kann.

Vergleicht man das Überleben von Nitrit-, TEModer EMS-behandeltem Kappa auf dem Wildtyp und hcr91, so ergibt sich mit allen drei Stoffen ein annähernd Dosis-unabhängiger Wirtszell-reaktivierbarer Anteil von $20-25 \%$ aller Letalschäden. Denselben Bruchteil findet man auch mit röntgenbestrahltem Kappa ${ }^{1}$. Diese Konstanz von 20-25\% könnte mit der Annahme erklärt werden, daß ein gleichhäufiger Anteil der Gesamtletalwirkung von Nitrit, TEM, EMS bzw. Röntgenstrahlen vielleicht auf Veränderungen der Sekundärstruktur der DNS beruht oder von solchen begleitet wird. Doch darf die Bedeutung dieser Konstanz nicht überbewertet werden, denn Röntgenstrahlen, Nitrit und EMS, vielleicht auch TEM, schädigen nicht nur die DNS, sondern auch das Protein von Phagen ${ }^{14-16}$. Somit überlebt ein gewisser Prozentsatz der Röntgen-bestrahlten oder chemisch behandelten Kappa-Phagen wahrscheinlich nur deshalb nicht, weil die an sich noch

14 P. Brookes U. P. D. Lawley, Biochem. J. 89, 138 [1963].

15 W. Harm, Z. Vererb.-Lehre 91, 52 [1960].

16 J. D. W überlebensfähige oder auch letal geschädigte, aber noch Wirtszell-reaktivierbare DNS nicht mehr in die Wirtszellen injiziert wird. Es ergibt sich daraus, daß die gefundenen Anteile Wirtszell-reaktivierbarer Letalschäden - basierend auf der Zählung von Plaques - nur Mindestwerte der Wirtszell-reaktivierbaren DNS-Schäden darstellen.

Extracellulär mit Hydroxylamin (HA) behandelter Kappa überlebt auf hcr42 und hcr64 ebenso gut wie auf dem Wildtyp von Serratia HY; nur auf hcr91 ist seine Chance zum Überleben um etwa $33 \%$ geringer (Tab. 1). Beim Testen weiterer 8 hoch UV-sensibler hor ${ }^{-}$-Mutanten mit HA-behandeltem Phagen wurde nur noch eine gefunden, die sich vom Wildtyp unterscheidet, und zwar in ähnlicher Weise wie hcr91. Unsere her -Mutanten sind also mit Hilfe von HA-behandeltem Kappa in zwei Gruppen einteilbar. Vielleicht repräsentieren diese Gruppen Defekte in zwei verschiedenen HCR-Mechanismen, die durch verschiedene Cistren gesteuert werden. Diese Hypothese ist nicht unwahrscheinlich; denn bei E. coli wurden schon drei im Genom unterschiedlich lokalisierte hcr-Gene durch Kreuzung gefunden ${ }^{17}$. Leider können bei Serratia HY noch nicht entsprechende Versuche gemacht werden, weil hier die Kreuzungs-Technik bisher unzureichend entwickelt ist.

Obwohl die beiden „HA-sensiblen“ SerratiaStämme vom Typ hcr9l verschiedenen Klonen entstammen, haben sie noch ein weiteres gemeinsames Merkmal, in dem sie sich vom Wildtyp und allen anderen 9 bisher untersuchten hcr-Mutanten unterscheiden: Nur in ihnen werden die durch extracelluläre UV-Bestrahlung in Kappa induzierten Prämutationen zum Klarplaque-Typ vollständig gelöscht ${ }^{18}$. Wahrscheinlich sind letztere Eigenschaft und die „HA-Sensibilität“ die pleiotropen Folgen ein und derselben Mutation.

\section{Diskussion}

Beim Studium der HCR interessiert besonders die Frage nach der chemischen Natur des oder der Wirtszell-reaktivierbaren DNS-Schäden. SAuERbIER fand, daß die Wirtszellreaktivierbarkeit der UV-bestrahlten Phagen T1 und P22 erlischt oder zumin-

17 J. Greenberg, Genetics 50, 639 [1964].

18 U. WINKLER, Z. Vererb.-Lehre, eingereicht. 
dest abnimmt, wenn in der Phagen-DNS das Thymin durch 5-Bromuracil substituiert worden ist ${ }^{19}$; er schreibt dazu: "it may be concluded that host ceil reactivable UV-lesions are located in or involve the thymine" 20. Seine Annahme wurde später durch andere Autoren gestützt ${ }^{3,6}$. Bei E. coli wurde nämlich gefunden, daß nur Zellen vom Wildtyp, nicht aber von den zugehörigen hcr ${ }^{-}$-Mutanten bei Bebrütung nach UV-Bestrahlung Oligonucleotide aus ihrer DNS heraustrennen können, die u. a. Thymin-Dimere enthalten.

In der vorliegenden Arbeit wurde nun gezeigt, $\mathrm{da} ß$ der Wildtyp von Serratia HY mindestens 20\% der durch Nitrit oder EMS im Phagen Kappa induzierten DNS-Letalschäden Wirtszell-reaktivieren kann. Dadurch wird es unwahrscheinlich, daß irgendwelche Thymin-Veränderungen eine notwendige Voraussetzung für die HCR sind; denn nach allgemeiner Kenntnis wird Thymin weder von Nitrit noch von EMS angegriffen.

Im Gegensatz zu unseren Befunden bei Kappa konnte Sauerbier nach Nitrit-Behandlung des E.coli. Phagen T1 und des Salmonella-Phagen P22 keine HCR nachweisen ${ }^{21}$. Sein negatives Resultat ist möglicherweise methodisch bedingt, denn er verwendete an Stelle von hcr--Mutanten nur hoch UV-bestrahlten Wildtyp. Es könnte aber auch sein, daß in E. coli B und Salmonella LT2 der HCR-Mechanismus nur eine geringere Anzahl von Schadensarten reparieren kann als derjenige in Serratia HY.

Bisher wurden insgesamt 7 Noxe ermittelt, deren Letalwirkung auf Kappa teilweise Wirtszell-reaktivierbar ist, nämlich UV und Röntgenstrahlen ${ }^{1}$, $p_{\mathrm{H}} 4,6$ bei $45^{\circ} \mathrm{C}$ (unveröff.), TEM, EMS, HA und Nitrit. Bei der Vielzahl und Unterschiedlichkeit der geprüften Noxe ist es unwahrscheinlich, daß sie alle in ihrer Art des Angriffes an der DNS-Primärstruk-

19 W. SAUerbier, Virology 15, 465 [1961].

20 W. Sauerbier, Z. Vererb.-Lehre 93, 220 [1962]. tur eine nicht seltene Gemeinsamkeit aufweisen, die der Angriffspunkt für die HCR sein könnte. Doch kann es sein, daß sich die gesuchte Gemeinsamkeit findet, wenn man die vielartigen Änderungen der Primärstruktur hinsichtlich ihrer häufigen Auswirkungen auf die Sekundärstruktur der DNS untersucht. Solche Auswirkungen bestehen in erster Linie in der Öffnung von Wasserstoff-Bindungen zwischen den komplementären Basen. Eine andere Möglichkeit könnte die Bildung kovalenter cross-links zwischen komplementären Strängen sein. Ob derartige Veränderungen an höheren Strukturen, wenn sie nicht nur kurze DNS-Abschnitte betreffen, sekundär die Spaltung von Phosphordiester-Bindungen zur Folge haben können, ist bisher unbekannt.

Eine genaue Definition der chemisch-strukturellen Voraussetzungen für das Wirksamwerden der HCR in geschädigter DNS ist gegenwärtig noch nicht möglich.

Die Versuche wurden durch eine Sachbeihilfe zum Habilitanden-Stipendium der Deutschen Fors c hungsgemeins ch a f t unterstützt. Das Triäthylenmelamin war ein Geschenk der Farbwerke Hoechst AG. Herrn Professor Dr. R. W. Kaplan danke ich für stetes Interesse und Frau Ingeborg Gieseke für gewissenhafte und fleißige Mitarbeit.

Anm. b. d. Korr.: Kurz vor und während der Drucklegung dieser Arbeit erschienen vier Veröffentlichungen anderer Autoren über die Wirtszellreaktivierung von chemisch induzierten Schäden in Bakterien-DNS; es wurden prinzipiell dieselben Schlußfolgerungen gezogen wie in der vorliegenden Arbeit über die Wirtszellreaktivierung chemisch geschädigter Phagen-DNS. R. P. Boyce u. P. Howard-Flanders, Z. Vererb.-Lehre 95, 345 [1964] ; B. Pairmeister u. C. L. Davison, Biochem. biophys. Res. Commun. 17, 608 [1964] ; P. C. Hanawalt u. R. H. Haynes, 19, 462 [1965]; K. W. Kohn, N. H. Steigbigel u. C. L. Spears, Proc. nat. Acad. Sci. USA 53, 1154 [1965].

21 W. SAUErbier, Virology 16, 398 [1962]. 